\title{
Critical Layer Analysis of Stuart Vortices in a Plane Jet
}

\author{
Ghada Alobaidi ${ }^{1}$ and Roland Mallier ${ }^{2}$ \\ ${ }^{1}$ Department of Mathematics and Statistics, American University of Sharjah, Sharjah, UAE \\ ${ }^{2}$ Department of Mathematics and Statistics, York University, Toronto, ON, Canada M3J 1P3 \\ Correspondence should be addressed to Ghada Alobaidi; galobaidi@aus.edu
}

Received 18 July 2013; Accepted 10 October 2013; Published 24 February 2014

Academic Editor: Mohamed Abd El Aziz

Copyright ( 2014 G. Alobaidi and R. Mallier. This is an open access article distributed under the Creative Commons Attribution License, which permits unrestricted use, distribution, and reproduction in any medium, provided the original work is properly cited.

Asymptotic techniques are used to model quasi-steady-state vortices in the plane (Bickley) inviscid jet. A nonlinear critical layer analysis is used to find a family of steady-state finite amplitude two-dimensional vortices which are based on the Stuart vortex.

\section{Introduction}

A very basic flow occurring in both nature and engineering applications is the wake behind a bluff body, and it has been claimed [1] that the plane (Bickley) jet, which has a $\operatorname{sech}^{2} y$ velocity profile, provides a very good approximation to such a wake. For this velocity profile, there exist both symmetric (sinuous) and antisymmetric (varicose) instability modes $[2,3]$ with critical layers centered on each of the two inflection points of the flow, as well as other modes with critical layers centered on the nose [4-6]. For twodimensional perturbations, Kelly [7] pointed out that since the wavenumber of the neutral varicose mode was onehalf that of the neutral sinuous mode, it was possible to have a resonant interaction between the two modes. Some experiments have hinted that an interaction may indeed take place but have not attempted to quantify it. The authors of [8] conducted careful experiments on small deficit (turbulent) wakes and found that the development of some aspects of the flow was dependent on initial conditions, which they attributed to interactions between the varicose and sinuous modes, and other experiments, such as $[9,10]$, have also suggested that these interactions may take place, as have preliminary numerical simulations [11]. Kelly [7] explored the idea of this interaction theoretically, using an unsteady viscous critical layer and assuming a modal interaction of the Stuart-Watson type with a cubic nonlinearity, but found that there was no interaction of this type because the coefficients of the coupling terms were identically zero. However, [12] employed instead an unsteady nonlinear critical layer, in which the nonlinear effects were confined to a thin layer in which the nonlinear and unsteady terms were of the same order of magnitude, and found that using this approach there was indeed a resonant interaction between the modes. The authors of [12] used an $\varepsilon^{1 / 2}$ critical layer, meaning that the width of the critical layer was $\mathcal{O}\left(\varepsilon^{1 / 2}\right)$, where $\varepsilon$ denoted the order of magnitude of the instability wave amplitude, and such a critical layer is standard for both steady $[13,14]$ and unsteady $[15,16]$ two-dimensional critical layer analyses and is also the scaling employed in the present study. For threedimensional disturbances, Mallier [17, 18] found that there was also an interaction between the varicose and sinuous modes.

As with any shear layer, when an instability mode is superimposed on a plane jet, the shear layer rolls up and a quasi-equilibrium state emerges in the form of large vortices. What is distinct about the plane jet is that two rows of vortices develop rather than just one, with a row centered on each of the critical layers. These large scale vortices are intriguing for a number of reasons, not least because it appears that an ordered state emerges from disorder, and the aim of the present study is to use asymptotic techniques to model them. Because the two instability modes can interact, our analysis must include both modes. 
For a mixing layer with a single row of vortices, a single critical layer, and a single instability mode, two alternative models emerged in the 1960s, Stuart vortices [19] and Benney-Bergeron-Davis (BBD) vortices [13, 14, 20-24]. Stuart found an exact nonlinear steady-state solution of the inviscid Euler equations, for which the stream function can be cast in the form $\psi=\ln [\cosh y-\varepsilon \cos x]$, where $\varepsilon$ is a small parameter. This solution represents an infinite row of corotating vortices, and when $\varepsilon= \pm 1$, the vortices become point vortices, but when $\varepsilon=0$, we recover unidirectional shear flow. In the form above, this stream function obeys $\nabla^{2} \psi=\left(1-\varepsilon^{2} \psi\right) e^{-2 \psi}$, which is a form of Liouville's equation. Since the vorticity is given by $\omega=-\nabla^{2} \psi$, the vorticity distribution for the Stuart vortex is

$$
\omega=-\frac{1-\varepsilon^{2}}{[\cosh y-\varepsilon \cos x]^{2}} .
$$

The stability of Stuart vortices has been studied both numerically and analytically [25-28]. By contrast, the BBD vortices, which marked the genesis of nonlinear critical layer theory, were found using matched asymptotic expansions, posing an outer solution away from the critical layer and matching it to an inner solution close to the critical layer. Recently, Caillol and coworkers have used an analysis similar to the BBD theory to model steady multipolar planar vortices [29] and also the intensification or weakening of a rapidly rotating vortex of a hurricane or tornado type by considering the critical-layer-like interaction between a helical neutral vortex Rossby wave and an azimuthally mean vortex [29-31]. For time-independent inviscid two-dimensional flow, it can be shown that the vorticity must be a function of the stream function, so that

$$
\nabla^{2} \psi=\mathscr{F}(\psi),
$$

with Liouville's equation being a well-known specific example and the sinh-Poisson equation [32] another. It has been shown [33] that there is no single-valued solution to (2) for the plane jet with critical layers centered on the inflection points, so we must seek a series solution. While the vorticity for the Stuart vortex was given by (1), the BBD theory instead invoked the Prandtl-Batchelor theorem [34], which says that, for steady flow at high Reynolds numbers, the vorticity distribution inside closed streamlines must be uniform. Because of this, BBD argued that the vorticity should be uniform inside the vortex cores, so that the function $\mathscr{F}$ was taken to be constant there.

One early problem with the BBD theory was that the vorticity was discontinuous across the edges of the cat's eyes, which BBD claimed would be smoothed out by a viscous layer at the edge of the cat's eyes. However, the analysis at the corners of the cat's eye led to a Wiener-Hopf problem, and Brown and Stewartson [35] later showed that this Wiener-Hopf problem did not have a solution with the correct behavior, meaning that the discontinuity could not be removed. Haberman $[23,24]$ showed that the problems with the BBD solutions could be removed if certain mean flow distortions were added to the problem, and we include those same mean flow corrections in our analysis here as series about the critical layers. In Haberman's solutions, the velocity and vorticity were continuous, but gradients of vorticity were not, and the same will hold true here, although it is possible to smooth out the discontinuity in the gradient of vorticity by utilizing a thin viscous layer at the edge of the vortex cores.

A second problem with the BBD solutions was also pointed out by Stewartson $[16,36]$. In order for vorticity to homogenize inside closed streamlines, as it is assumed to do in the BBD solution where the Prandtl-Batchelor theorem was invoked, some viscosity must be present. Stewartson argued that the cat's eyes would decay before the vorticity had become fully homogenized, and indeed it is known that such a situation occurs for the Blasius boundary layer [37, 38]. However, since structures resembling the BBD solutions were reported in simulations such as those of [39], it was widely assumed that the BBD solutions must be correct. Klaassen and Peltier [40] later pointed out that the cat's eyes structures observed in simulations also resembled Stuart vortices, and this can be seen upon close examination of the original paper of [39]. Although the vorticity contours in [39] resemble both the BBD solutions and Stuart vortices, the authors describe the "topography" of the vorticity field as consisting of a high rounded hill (the core) along with high ridges (the braids), with the core and braids separated by low passes. It is this hill-like structure of the vorticity inside the core, which the authors of [39] attribute to the coalescence of the vorticity into relatively compact cores, that suggests that the flow inside the core is the Stuart vortex rather than the BBD solutions, for which the vorticity field would be a plateau rather than a round hill. Similar results can be found in the simulations of [41] that calculated the quasi-equilibrium states in order to study their stability. In their Figure 3, they present surface plots of the vorticity field. As in [39], the vorticity field consists of a smooth round hill (the core) along with high ridges (the braids), with the two separated by a trough, which they attribute to the entrainment of irrotational fluid into the core.

In later simulations, Sommeria et al. [42] confirmed that the vorticity field in the quasi-equilibrium states resembled the Stuart vortex rather than the homogenized cores of the $\mathrm{BBD}$ solutions and suggested that the Stuart vortex was a preferred state on entropy grounds. In their Figures 2-4, they show the rollup of vorticity. Because their contours are labelled, it is possible to discern from their simulations that after the climax, the vorticity decays to a smooth state resembling a round hill. In their Figures 8-10, the authors of [42] plot a scatter-plot of the vorticity against the stream function for the steady state. If the vorticity distribution was exactly that of the Stuart vortex, it would obey Liouville's equation, and a plot of the log of the vorticity against the stream function should be a straight line. The authors of [42] claim that, at high Reynolds numbers, inside the core there is excellent agreement between their scatter-plots and a straight line, so that the vorticity distribution in the equilibrium states is the Stuart vortex.

Finally, Wang and Maxey [43] gave a kinematical description of the mixing process. They noted that, in the quasisteady state, the vorticity field develops into a well-defined 
structure similar to the Stuart vortex. In their Figure 1, they plot contours of vorticity, and their use of color in this figure enables the structure of the vorticity field to be clearly seen, with once again the vorticity developing into a round hill surrounded by the ridge-like braids. Wang and Maxey attributed this to the vorticity layer being stretched and wrapped into a spiral structure and then diffusion acting to smooth the vorticity in between the spirals, and they show a cartoon of this process in their Figure 4.

The simulations discussed above suggest that, rather than the vorticity homogenizing inside the vortex cores, an alternative scenario, presented by Rhines and Young [44] in a different context, occurs. Rhines and Young argued that homogenization of vorticity within closed streamlines will take place in two stages: a rapid phase dominated by shearaugmented diffusion in which the vorticity will tend to a smooth but not necessarily uniform distribution and a second much slower phase in which the process of homogenization will be completed. The simulations presented in [42] suggest that the first, rapid, phase postulated by Rhines and Young will take place during vortex rollup in a shear layer but that, as suggested by Stewartson, the vortices will decay before the second stage has occurred, so that the second stage would only occur if forcing were present. Thus, the vorticity inside the vortex cores will have a smooth but nonuniform distribution, and we believe, on entropy grounds, that the Stuart vortex is that distribution. These same arguments apply to the vorticity distribution inside the vortex cores of the plane jet considered here.

In our study, we have modeled a quasi-steady state with a steady state, and, as pointed out by a referee, a steady state may not in fact be achievable in practice. The viscosity needed to smooth the vorticity distribution inside the core would ultimately cause the cat's eye to decay, and we are modeling the quasi-steady state which develops before this decay sets in. The unattainability of a steady state is a longstanding concern about vortex solutions; for example, [45-47] would seem to indicate that the flow in the unsteady twodimensional critical layer analyses of $[15,16]$ would never reach a steady state.

Caillol and Grimshaw have considered the unattainability of a steady state in some detail, and they invoked the PrandtlBatchelor theorem [34] and the work of Rhines and Young [44] and extended it for the cases they studied. Caillol and Grimshaw considered several quasi-steady regimes with critical layers, including an internal solitary wave with a weakly stratified critical layer [48], a Rossby solitary wave [49], a Rossby elevation wave where no induced mean flow is created [50], a finite-period neutral shear wave in a vortex [30], and multipolar planar vortices [31]. In their studies, Caillol and Grimshaw drew on earlier work by Troitskaya and collaborators in Nizhny Novgorod on the subtle role of viscosity in nonlinear critical layers $[51,52]$.

In the present study, we will use a time-independent nonlinear critical layer analysis to model the vortices which develop during the rollup of a plane jet, using asymptotic techniques due to $[13,14]$ but with a vorticity distribution inside the cores which is smooth but nonconstant, resembling Stuart vortices [19] rather than BBD vortices, and in that sense our solution may be thought of as Stuart vortices in a plane jet. This sort of approach has been used previously both by ourselves for rotating and stratified mixing layers [53-55] and by others $[33,56]$. By contrast, compressible Stuart vortices were found numerically $[57,58]$. The difference between the plane jet discussed here and the mixing layer is that the plane jet has two critical layers, with a separate expansion required for each of these critical layers, and we must include both the varicose and sinuous modes in these expansions.

The rest of the paper is as follows. In Section 2, we present our analysis and find the steady-state vortex solutions. Essentially, our analysis involves posing an outer expansion in the main body of the fluid away from the critical layer, at which the base velocity is equal to the phase velocity of the disturbance, and an inner expansion near to the critical layer, using rescaled variables, and matching the two expansions together. For the outer expansion, we use the inviscid incompressible Euler equations in the stream function formulation. We suppose that we have superimposed a small, spatially periodic disturbance on the base flow and expand in powers of the amplitude; this expansion becomes disordered at the critical layer where it is necessary to pose an inner expansion using stretched coordinates. In this inner solution, rather than assuming that vorticity is uniform (homogenized) inside the closed cat's eyes (as was done in the BBD theory and its extensions by Maslowe and Haberman), we use the Stuart vortex as our guide, for the reasons outlined above. Finally, in Section 3, we will make some concluding remarks.

\section{Analysis}

2.1. Formulation and Outer Expansion. The motion of an inviscid incompressible fluid in two dimensions is governed by the Euler equations in the vorticity-stream function formulation:

$$
\nabla^{2} \psi_{t}-\frac{\partial\left(\psi, \nabla^{2} \psi\right)}{\partial(x, y)}=0,
$$

where $\psi(x, y, t)$ is the stream function, with the velocity given by $\underline{u}=\left(\psi_{y},-\psi_{x}, 0\right)$. The objective of the present study is to seek nonlinear waves of permanent form which are periodic in $\xi=\alpha(x-c t)$, where $\alpha$ is the wavenumber and $c$ is the phase velocity. If we work in a frame traveling in the streamwise direction at the phase velocity, we can set $c=0$ without affecting our analysis, so that $\xi=\alpha x$ and our solutions are independent of time, and we can replace (3) by

$$
\frac{\partial\left(\psi, \nabla^{2} \psi\right)}{\partial(x, y)}=0,
$$

which has a solution of the functional form

$$
\nabla^{2} \psi=\mathscr{F}(\psi) \text {. }
$$

To find our solution, we will pose a weakly nonlinear expansion of the form

$$
\begin{aligned}
\psi= & \psi^{(0)}+\varepsilon\left[\phi_{1}^{(1)} e^{i \xi}+\phi_{2}^{(1)} e^{2 i \xi}+c c\right] \\
& +\varepsilon^{2}\left[\phi_{0}^{(2)}+\left(\phi_{1}^{(2)} e^{i \xi}+\phi_{2}^{(2)} e^{2 i \xi}\right.\right.
\end{aligned}
$$




$$
\begin{gathered}
\left.\left.+\phi_{3}^{(2)} e^{3 i \xi}+\phi_{4}^{(2)} e^{4 i \xi}+\mathrm{cc}\right)\right] \\
+\varepsilon^{3}\left[\phi_{0}^{(3)}+\left(\phi_{1}^{(3)} e^{i \xi}+\phi_{2}^{(3)} e^{2 i \xi}+\phi_{3}^{(3)} e^{3 i \xi}+\phi_{4}^{(3)} e^{4 i \xi}\right.\right. \\
\left.\left.+\phi_{5}^{(3)} e^{5 i \xi}+\phi_{6}^{(3)} e^{6 i \xi}+\mathrm{cc}\right)\right]+\cdots
\end{gathered}
$$

where cc means complex conjugate and $\varepsilon \sim o(1)$ is a scaling parameter, while $\psi^{(0)}=\tanh y-2 y / 3$ is the base flow which is related to the base velocity $u^{(0)}=\operatorname{sech}^{2} y-2 / 3$ by $\psi^{(0) \prime}(y)=$ $u^{(0)}(y)$. The " $2 / 3$ " here is the well-known value of the phase velocity for singular neutral modes which have critical layers at the inflection points of the flow, that is, at $y= \pm y_{c}$, where $y_{c}=\operatorname{arccosh} \sqrt{3 / 2}$ with $u^{(0)}\left( \pm y_{c}\right)=u^{(0) \prime \prime}\left( \pm y_{c}\right)=0$. There are two such modes, a varicose mode with a streamwise wavenumber of 1 and a sinuous mode with a streamwise wavenumber of $2[2,3]$. It has been shown [33] that there is no single-valued solution to (5) for the Bickley jet with this phase velocity; that is why we must seek a series solution. We will also expand the wavenumber $\alpha=1+\varepsilon \alpha_{1}+\cdots$ to allow for a small amount of detuning. By substituting expansion (6) into governing equation (4), we get a series of equations at successive powers of $\varepsilon$, and to abbreviate these equations, we introduce the operator $\mathscr{L}_{n} \equiv\left(\partial^{2} / \partial y^{2}\right)-\left(u_{0}^{\prime \prime} / u_{0}\right)-n^{2}$ and the functions $f_{1}=\left(u_{0}^{\prime \prime \prime} / u_{0}^{2}\right)-\left(u_{0}^{\prime} u_{0}^{\prime \prime} / u_{0}^{3}\right), f_{2}=\left(u_{0}^{\prime \prime \prime \prime} / 6 u_{0}^{3}\right)-$ $\left(u_{0}^{\prime \prime 2} / 6 u_{0}^{4}\right)-\left(u_{0}^{\prime} u_{0}^{\prime \prime \prime} / 2 u_{0}^{4}\right)+\left(u_{0}^{\prime 2} u_{0}^{\prime \prime} / 2 u_{0}^{3}\right)$, and $f_{3}=\left(\phi_{0}^{(2) \prime \prime \prime} / u_{0}\right)-$ $\left(\phi_{0}^{(2) \prime \prime} u_{0}^{\prime \prime} / u_{0}^{2}\right)$.

At leading order, $\mathcal{O}(\varepsilon)$, we assume that the disturbance is comprised of two modes, which obey $\mathscr{L}_{1} \phi_{1}^{(1)}=0$ and $\mathscr{L}_{2} \phi_{2}^{(1)}=0$. There are well-known closed form solutions $\phi_{1}^{(1)}=A_{1} \operatorname{sech} y \tanh y$ and $\phi_{2}^{(1)}=A_{2} \operatorname{sech}^{2} y$ for these two modes $[2,3]$, where the constants $A_{1}$ and $A_{2}$ are a measure of the relative magnitudes of the wavenumbers 1 and 2 modes. We need to expand these solutions as series about the critical layers at $y= \pm y_{c}$, using the variables $y_{ \pm}=y \mp y_{c}$, as follows:

$$
\begin{gathered}
\phi_{1}^{(1)} \sim \pm\left[1 \pm \frac{y_{+}}{\sqrt{3}}-\frac{3 y_{+}^{2}}{2} \pm \frac{5 y_{+}^{3}}{6 \sqrt{3}}+\frac{43 y_{+}^{4}}{72}+\cdots\right] \frac{2^{1 / 2} A_{1}}{3}, \\
\phi_{2}^{(1)} \sim\left[1 \mp \frac{2 y_{+}}{\sqrt{3}} \pm \frac{4 y_{+}^{3}}{3 \sqrt{3}}-\frac{4 y_{+}^{4}}{9}+\cdots\right] \frac{2 A_{2}}{3},
\end{gathered}
$$

where we take the upper and lower elements in \pm and $\mp$ for the expansion about $y=y_{c}$ and $y=-y_{c}$, respectively.

At the next order, $\mathcal{O}\left(\varepsilon^{2}\right)$, we need to include a mean flow distortion term, $\phi_{0}^{(2)} \sim \phi_{0 c}^{(2) \pm}+\phi_{0 c}^{(2) \pm \prime} y_{ \pm}+\left(\phi_{0 c}^{(2) \pm \prime \prime} y_{ \pm}^{2} / 2 !\right)+\cdots$, and we need to calculate four modes:

$$
\begin{gathered}
\mathscr{L}_{1} \phi_{1}^{(2)}=2 \alpha_{1} \phi_{1}^{(1)}+f_{1} \phi_{1}^{(1)} \phi_{2}^{(1)}, \\
\mathscr{L}_{2} \phi_{2}^{(2)}=8 \alpha_{1} \phi_{2}^{(1)}+\frac{f_{1} \phi_{1}^{(1) 2}}{2}, \\
\mathscr{L}_{3} \phi_{3}^{(2)}=f_{1} \phi_{1}^{(1)} \phi_{2}^{(1)}, \\
\mathscr{L}_{4} \phi_{4}^{(2)}=\frac{f_{1} \phi_{2}^{(1) 2}}{2},
\end{gathered}
$$

which we can solve as series about the critical layers:

$$
\begin{aligned}
\phi_{1}^{(2)} \sim & B_{1}^{(2) \pm} \mp \frac{2^{3 / 2} A_{1} A_{2} y_{ \pm} \ln y_{ \pm}}{3}+C_{1}^{(2) \pm} y_{ \pm} \\
& +\left( \pm \frac{2^{1 / 2} \alpha_{1} A_{1}}{3}-\frac{3 B_{1}^{(2) \pm}}{2}+\frac{2^{3 / 2} A_{1} A_{2}}{3^{3 / 2}}\right) \\
& \times y_{ \pm}^{2}+\cdots, \\
\phi_{2}^{(2)} \sim & B_{2}^{(2) \pm}-\frac{2 A_{1}^{2} y_{ \pm} \ln y_{ \pm}}{3}+C_{2}^{(2) \pm} y_{ \pm} \\
& +\left(\frac{8 \alpha_{1} A_{2}}{3} \mp \frac{2 A_{1}^{2}}{3^{3 / 2}}\right) y_{ \pm}^{2}+\cdots \\
\phi_{3}^{(2)} \sim & B_{3}^{(2) \pm} \mp \frac{2^{5 / 2} A_{1} A_{2} y_{ \pm} \ln y_{ \pm}}{3}+C_{3}^{(2) \pm} y_{ \pm} \\
& +\left(\frac{5 B_{3}^{(2) \pm}}{2}+\frac{2^{3 / 2} A_{1} A_{2}}{3^{3 / 2}}\right) y_{ \pm}^{2}+\cdots, \\
& +\left(6 B_{4}^{(2) \pm} \pm \frac{8 A_{2}^{2}}{3^{3 / 2}}\right) y_{ \pm}^{2}+\cdots, \\
\phi_{4}^{(2)} \sim & B_{4}^{(2) \pm}-\frac{4 A_{2}^{2} y_{ \pm} \ln y_{ \pm}}{3}+C_{4}^{(2) \pm} y_{ \pm} \\
& \left(\frac{1}{3}\right)
\end{aligned}
$$

where the B's and C's are constants which can be evaluated by enforcing the condition that the $\phi$ 's vanish as $y \rightarrow \pm \infty$.

At $\mathcal{O}\left(\varepsilon^{3}\right)$, once again we need a mean flow distortion term, which at this order is singular at the critical layers, $\phi_{0}^{(3)} \sim$ $\left(\phi_{0 c,-1}^{(3) \pm} / y_{ \pm}\right)+\phi_{0 c}^{(3) \pm}+\phi_{0 c}^{(3) \pm \prime} y_{ \pm}+\left(\phi_{0 c}^{(3) \pm \prime \prime} y_{ \pm}^{2} / 2 !\right)+\cdots$, and we need to calculate six modes:

$$
\begin{gathered}
\mathscr{L}_{1} \phi_{1}^{(3)}=2 \alpha_{1} \phi_{1}^{(2)}+\left(\alpha_{1}^{2}+2 \alpha_{2}+f_{3}\right) \phi_{1}^{(1)}-3 f_{2} \phi_{1}^{(1) 3} \\
+f_{1}\left(\phi_{1}^{(1)} \phi_{2}^{(2)}+\phi_{2}^{(1)} \phi_{1}^{(2)}+\phi_{2}^{(1)} \phi_{3}^{(2)}\right. \\
\left.-\frac{2 \phi_{1}^{(1)}\left(\phi_{1}^{(1)} \phi_{1}^{(1) \prime}+\phi_{2}^{(1)} \phi_{2}^{(1) \prime}\right)}{u_{0}}\right) \\
\mathscr{L}_{2} \phi_{2}^{(3)}=8 \alpha_{1} \phi_{2}^{(2)}+\left(4 \alpha_{1}^{2}+8 \alpha_{2}+f_{3}\right) \phi_{2}^{(1)}-3 f_{2} \phi_{2}^{(1) 3} \\
+f_{1}\left(\phi_{1}^{(1)} \phi_{1}^{(2)}+\phi_{2}^{(1)} \phi_{4}^{(2)}+\phi_{1}^{(1)} \phi_{3}^{(2)}\right. \\
\left.-\frac{2 \phi_{2}^{(1)}\left(\phi_{1}^{(1) \prime} \phi_{2}^{(1)}+\phi_{1}^{(1)} \phi_{2}^{(1) \prime}\right)}{u_{0}}\right) \\
+3 f_{2} \phi_{1}^{(1) 2} \phi_{2}^{(1)}, \\
\mathscr{L}_{3} \phi_{3}^{(3)}=18 \alpha_{1} \phi_{3}^{(2)}+f_{1}\left(\phi_{1}^{(1)} \phi_{2}^{(2)}+\phi_{2}^{(1)} \phi_{1}^{(2)}+\phi_{1}^{(1)} \phi_{2}^{(2)}\right) \\
+f_{2}\left(\phi_{1}^{(1) 3}+3 \phi_{1}^{(1)} \phi_{2}^{(1) 2}\right) \\
\mathscr{L}_{4} \phi_{4}^{(3)}=32 \alpha_{1} \phi_{4}^{(2)}+f_{1}\left(\phi_{1}^{(1)} \phi_{3}^{(2)}+\phi_{2}^{(1)} \phi_{2}^{(2)}\right)
\end{gathered}
$$




$$
\begin{aligned}
\mathscr{L}_{5} \phi_{5}^{(3)}= & f_{1}\left(\phi_{1}^{(1)} \phi_{4}^{(2)}+\phi_{2}^{(1)} \phi_{3}^{(2)}\right)+3 f_{2} \phi_{1}^{(1)} \phi_{2}^{(1) 2}, \\
& \mathscr{L}_{6} \phi_{6}^{(3)}=f_{1} \phi_{2}^{(1)} \phi_{4}^{(2)}+f_{2} \phi_{2}^{(1) 3},
\end{aligned}
$$

which we can again solve as series:

$$
\begin{gathered}
\phi_{1}^{(3)} \sim \frac{A_{1}^{3}}{2^{3 / 2} 3^{1 / 2} y_{ \pm}} \pm\left(\frac{A_{1}^{2}}{2^{1 / 2} 3}-\frac{2^{7 / 2} A_{2}^{2}}{3}\right) A_{1} \ln y_{ \pm}+B_{1}^{(3) \pm}, \\
\phi_{2}^{(3)} \sim \pm \frac{A_{2}^{3}}{3^{1 / 2} y_{ \pm}}+\left(-\frac{4 A_{2}^{2}}{3}+\frac{4 A_{1}^{2}}{3}\right) A_{2} \ln y_{ \pm}+B_{2}^{(3) \pm} \\
\phi_{3}^{(3)} \sim-\left(\frac{A_{1}^{2}}{2^{3 / 2} 3^{3 / 2}}+\frac{A_{2}^{2}}{2^{1 / 2} 3^{1 / 2}}\right) \frac{A_{1}}{y_{ \pm}} \\
\pm\left(\frac{A_{1}^{2}}{2^{1 / 2} 3}-2^{1 / 2} A_{2}^{2}\right) A_{1} \ln y_{ \pm}+B_{3}^{(3) \pm} \\
\phi_{4}^{(3)} \sim \mp \frac{A_{1}^{2} A_{2}}{3^{1 / 2} 2 y_{ \pm}}+B_{4}^{(3) \pm} \\
\phi_{5}^{(3)} \sim-\frac{A_{1} A_{2}^{2}}{6^{1 / 2} y_{ \pm}}+B_{5}^{(3) \pm} \mp 2^{1 / 2} A_{1} A_{2}^{2} \ln y_{ \pm} \\
\phi_{6}^{(3)} \sim \mp \frac{A_{2}^{3}}{3^{3 / 2} y_{ \pm}}+B_{6}^{(3) \pm}-\frac{4 A_{2}^{3} \ln y_{ \pm}}{3} .
\end{gathered}
$$

These solutions and their derivatives are singular in the limit $y_{+} \rightarrow 0$, with these discontinuities being caused by the expansion becoming disordered near the critical layers, and to remedy this, it is necessary to use stretched variables in the critical layers.

2.2. Inner Expansion. To remedy the singularities observed in the outer expansion, we will follow $[13,14]$ and introduce the stretched variables $y_{+}=y-y_{c}=\varepsilon^{1 / 2} Y_{+}, y_{-}=y+y_{c}=$ $\varepsilon^{1 / 2} Y_{-}$, and $\psi=\varepsilon \Psi$ in the critical layers. This scaling is chosen so that nonlinear terms enter at the same order as the singularities inside the critical layer. Once again, we will pose an expansion in $\varepsilon$, the form of which is suggested by the outer solution rewritten in the inner variables as follows:

$$
\begin{aligned}
\Psi \sim & \frac{\Psi^{(-2)}}{\varepsilon}+\Psi^{(0)}+\varepsilon^{1 / 2} \Psi^{(1)}+\varepsilon \Psi^{(2)} \\
& +\varepsilon^{3 / 2}\left[\Psi^{(3)}+\left(\ln \varepsilon^{1 / 2}\right) \Psi^{(3 l)}\right]+\cdots,
\end{aligned}
$$

with the $\varepsilon^{3 / 2}\left(\ln \varepsilon^{1 / 2}\right)$ terms caused by the logs in the outer solution. We will have slightly different solutions for the expansions inside the upper and lower critical layers. In what follows, we will use the superscripts "+" and "-" to denote the solutions at the upper and lower critical layers, respectively, and we will drop the superscript when the two expressions are the same.

At $\mathcal{O}\left(\varepsilon^{-1}\right)$, we have a constant term, $\Psi^{(-2) \pm}= \pm 3^{-1 / 2} \mp$ $2 y_{c} / 3$.
At $\mathcal{O}\left(\varepsilon^{0}\right)$, our solution must obey

$$
\frac{\partial\left(\Psi^{(0)}, \Psi_{Y Y}^{(0)}\right)}{\partial(\xi, Y)}=0
$$

which has a general solution $\Psi_{Y_{ \pm} Y_{ \pm}}^{(0) \pm}=\mathscr{F}^{(0) \pm}\left(\Psi^{(0) \pm}\right)$. In order to solve (13) and subsequent equations, we will use the outer solution as our guide. At this order, the outer solution written in inner variables becomes

$$
\Psi^{(0) \pm}=\mp \frac{2 Y_{ \pm}^{2}}{3^{3 / 2}}+\left[\frac{2 A_{2} e^{2 i \xi}}{3} \pm \frac{2^{1 / 2} A_{1} e^{i \xi}}{3}+\mathrm{cc}\right],
$$

and since this is nonsingular and satisfies (13), we will use it as our inner solution. Solution (14) is of the same form as the leading order terms of both the Stuart vortex stream function [19] and the BBD solution but with of course two waves present rather than just one. Using (14), the $\mathcal{O}\left(\varepsilon^{1 / 2}\right)$ inner equation is

$$
\frac{\partial\left(\Psi^{(0)}, \Psi_{Y Y}^{(1)}\right)}{\partial(\xi, Y)}=0
$$

and once again, our outer solution written in inner variables satisfies this and is nonsingular, so we may use it as our solution:

$$
\Psi^{(1) \pm}=\mp \frac{4 A_{2} e^{2 i \xi}}{3^{3 / 2}}+\frac{2^{1 / 2} A_{1} e^{i \xi}}{3^{3 / 2}}+\mathrm{cc} .
$$

Using (14) and (16), the $\mathcal{O}(\varepsilon)$ inner equation is

$$
\frac{\partial\left(\Psi^{(0)}, \Psi_{Y Y}^{(2)}\right)}{\partial(\xi, Y)}=\Psi_{Y}^{(0)} \Psi_{\xi \xi \xi}^{(0)},
$$

and once again, our outer solution written in inner variables satisfies this and is nonsingular, so we may use it as our solution as follows:

$$
\begin{aligned}
\Psi^{(2) \pm}= & \frac{2 Y_{ \pm}^{4}}{3^{5 / 2}}+\phi_{0 c}^{(2) \pm} \\
& +\left[B_{4}^{(2) \pm} e^{4 i \xi}+B_{3}^{(2) \pm} e^{3 i \xi}+B_{2}^{(2) \pm} e^{2 i \xi}\right. \\
& \left.+\left(B_{1}^{(2) \pm} \mp \frac{A_{1} Y_{+}^{2}}{2^{1 / 2}}\right) e^{i \xi}+c c\right] .
\end{aligned}
$$

At the first logarithmic order $\mathcal{O}\left(\varepsilon^{3 / 2} \ln \left(\varepsilon^{1 / 2}\right)\right)$, the inner equation is

$$
\frac{\partial\left(\Psi^{(0)}, \Psi_{Y Y}^{(3 l)}\right)}{\partial(\xi, Y)}=0
$$

and we may again use the outer expansion to construct our inner solution as follows:

$$
\begin{aligned}
& \Psi^{(3 l) \pm}=-\frac{Y_{ \pm}}{3}\left[4 A_{2}^{2} e^{4 i \xi} \pm 2^{5 / 2} A_{1} A_{2}\left(e^{3 i \xi}+e^{i \xi}\right)\right. \\
& \left.+2 A_{1}^{2} e^{2 i \xi}+\mathrm{cc}\right] \text {. }
\end{aligned}
$$


At $\mathcal{O}\left(\varepsilon^{3 / 2}\right)$, the inner equation is

$$
\begin{aligned}
\frac{\partial\left(\Psi^{(0)}, \Psi_{Y Y}^{(3)}\right)}{\partial(\xi, Y)} & +\frac{\partial\left(\Psi^{(1)}, \Psi_{Y Y}^{(2)}\right)}{\partial(\xi, Y)}+\frac{\partial\left(\Psi^{(0)}, \Psi_{\xi \xi}^{(1)}\right)}{\partial(\xi, Y)} \\
& -\Psi_{\xi \xi \xi}^{(0)} \Psi_{Y}^{(1)}=0 .
\end{aligned}
$$

It is at this order that the singularities appear in the outer solution written in inner variables, and as $Y_{ \pm} \rightarrow \pm \infty$, we have

$$
\begin{aligned}
\Psi^{(3 \pm)}= & -\frac{8 Y_{ \pm}^{5}}{135}+\phi_{0 c}^{(2) \pm \prime} Y_{ \pm}+\frac{\phi_{0 c,-1}^{(3) \pm}}{Y_{ \pm}} \\
& +\left[\frac{5 A_{1} Y_{ \pm}^{3}}{3^{5 / 2} 2^{1 / 2}}+C_{1}^{(2) \pm} Y_{ \pm} \mp \frac{2^{5 / 2} A_{1} A_{2} Y_{ \pm} \ln Y_{ \pm}}{3}\right. \\
& \left.+\frac{A_{1}^{3}}{2^{3 / 2} 3^{1 / 2} Y_{ \pm}}\right]\left(e^{i \xi}+e^{-i \xi}\right) \\
& +\left[ \pm \frac{8 A_{2} Y_{ \pm}^{3}}{3^{5 / 2}}+C_{2}^{(2) \pm} Y_{ \pm}-\frac{2 A_{1}^{2} Y_{ \pm} \ln Y_{ \pm}}{3} \pm \frac{A_{2}^{3}}{3^{1 / 2} Y_{ \pm}}\right] \\
& \times\left(e^{2 i \xi}+e^{-2 i \xi}\right) \\
& +\left[C_{3}^{(2) \pm} Y_{ \pm} \mp \frac{2^{5 / 2} A_{1} A_{2}}{3} Y_{ \pm} \ln Y_{ \pm}\right. \\
& -\frac{A_{1} A_{2}^{2}}{6^{1 / 2} Y_{ \pm}}\left(e^{5 i \xi}+e^{-5 i \xi}\right) \mp \frac{A_{2}^{3}}{3^{3 / 2} Y_{ \pm}}\left(e^{6 i \xi}+e^{-6 i \xi}\right) \\
& +\left[C_{4}^{(2) \pm} Y_{ \pm}-\frac{4 A_{2}^{2}}{3} Y_{ \pm} \ln Y_{ \pm} \mp \frac{A_{1}^{2} A_{2}}{3^{1 / 2} 2 Y_{ \pm}}\right] \\
& \times\left(e^{4 i \xi}+e^{-4 i \xi}\right) \\
6^{1 / 2} & \left.\left.\frac{A_{1}^{2}}{6^{3 / 2}}\right) \frac{A_{1}}{Y_{ \pm}}\right]
\end{aligned}
$$

To proceed, we must solve for the flow inside and outside the core separately. Inside the core, we can assume that the solution is regular and take $\Psi^{(3)}=\Psi^{(3 C)}$ with

$$
\begin{aligned}
\Psi^{(3 C \pm)}= & \phi_{0 c}^{(2) \pm \prime} Y_{ \pm}+\left[\left(-\frac{A_{1} Y_{ \pm}^{3}}{3^{3 / 2} 2^{1 / 2}}+C_{1}^{(2) \pm} Y_{ \pm}\right) e^{i \xi}\right. \\
& \left.+\left(C_{2}^{(2) \pm} e^{2 i \xi}+C_{3}^{(2) \pm} e^{3 i \xi}+C_{4}^{(2) \pm} e^{4 i \xi}\right) Y_{ \pm}+c c\right]
\end{aligned}
$$

which satisfies (21), while outside the core we must take $\Psi^{(3)}=$ $\Psi^{(3 C)}+\Psi^{(3 D)}$ with

$$
\frac{\partial\left(\Psi^{(0)}, \Psi_{Y Y}^{(3 D)}\right)}{\partial(\xi, Y)}=0
$$

which has a general solution $\Psi_{Y_{ \pm} Y_{ \pm}}^{(3 D) \pm}=\mathscr{F}^{(3) \pm}\left(\Psi^{(0) \pm}\right)$. From the outer expansion, we can deduce

$$
\begin{aligned}
\mathscr{F}^{(3) \pm} \sim \gamma[ & \frac{2^{7 / 2}}{3^{3 / 4}}\left(\mp \Psi^{(0) \pm}\right)^{3 / 2}-3^{-7 / 4} \\
& \times\left(2^{5 / 2} A_{1}^{2}+2^{7 / 2} A_{2}^{2}\right)\left(\mp \Psi^{(0) \pm}\right)^{-1 / 2} \\
& -2^{5 / 2}\left(3^{-9 / 4} \psi_{0 c,-1}^{(3)+} \pm 3^{-11 / 4} A_{1}^{2} A_{2}\right) \\
& \left.\times\left(\mp \Psi^{(0) \pm}\right)^{-3 / 2}+\cdots\right],
\end{aligned}
$$

where $\gamma=-1$ above the critical layer and +1 below it. To find the functional form of $\mathscr{F}^{(3)}$, we need to follow $[13,14]$ and reintroduce weak viscosity, which will allow us to obtain a viscous secularity condition. The viscous counterpart of (4) is

$$
\frac{\partial\left(\psi, \nabla^{2} \psi\right)}{\partial(\xi, y)}=-\frac{1}{\alpha \operatorname{Re}} \nabla^{4} \psi
$$

where Re is the Reynolds number. To ensure that viscous terms enter into the critical layer at the correct order, we will write $(\alpha \mathrm{Re})^{-1}=\varepsilon^{3 / 2} \lambda$, where $\lambda$ is the so-called Benney-Bergeron parameter $[13,14]$ measuring the relative importance of the viscous and nonlinear terms inside the critical layer. In our analysis, we will assume that viscosity is weak even inside the critical layer with $\lambda \ll 1$. If we denote the inner viscous solution by $\Psi^{(v)}$, we may expand this as a series in $\lambda$ :

$$
\Psi^{(v)} \sim \Psi+\lambda \widetilde{\Psi}+\mathcal{O}\left(\lambda^{2}\right)
$$

where the first term in the expansion is the inviscid solution and the second term is the viscous correction. We may also expand this viscous correction $\widetilde{\Psi}$ as a series in $\varepsilon$, just as we posed an expansion (12) of the inviscid solution $\Psi$. To determine the function $\mathscr{F}^{(3)}$, we need to arrive at an equation for the viscous correction to $\Psi^{(3 D)}$, but to do this, we first need to calculate the viscous correction at lower powers of $\varepsilon$. At the first few orders, we find

$$
\frac{\partial\left(\Psi^{(0)}, \widetilde{\Psi}_{Y Y}^{(0)}\right)}{\partial(\xi, Y)}=\frac{\partial\left(\Psi^{(0)}, \widetilde{\Psi}_{Y Y}^{(1)}\right)}{\partial(\xi, Y)}=\frac{\partial\left(\Psi^{(0)}, \widetilde{\Psi}_{Y Y}^{(2)}\right)}{\partial(\xi, Y)}=0,
$$

and the $\mathcal{O}\left(\varepsilon^{0}\right), \mathcal{O}\left(\varepsilon^{1 / 2}\right)$, and $\mathcal{O}\left(\varepsilon^{1}\right)$ viscous corrections all vanish, $\widetilde{\Psi}^{(0)}=\widetilde{\Psi}^{(1)}=\widetilde{\Psi}^{(2)}=0$. At $\mathcal{O}\left(\varepsilon^{3 / 2}\right)$, we find that $\widetilde{\Psi}^{(3 D)}$ obeys

$$
\frac{\partial\left(\Psi^{(0)}, \widetilde{\Psi}_{Y Y}^{(3 D)}\right)}{\partial(\xi, Y)}=-\Psi_{Y Y Y Y}^{(3 D)}=-\mathscr{F}^{(3)}\left(\Psi^{(0)}\right)_{Y Y} .
$$

If we use $\eta=\xi$ and $\Psi^{(0)}$ as von Mises coordinates, we can write (29) in the form

$$
\frac{\partial}{\partial \eta}\left(\widetilde{\Psi}_{Y Y}^{(3)}\right)=\frac{\partial}{\partial \Psi^{(0)}}\left[\Psi_{Y}^{(0)} \mathscr{F}^{(3) \prime}\left(\Psi^{(0)}\right)\right],
$$


which can be integrated with respect to $\eta$ to give

$$
\widetilde{\Psi}_{Y Y}^{(3)}=\frac{\partial}{\partial \Psi^{(0)}}\left[\mathscr{F}^{(3) \prime}\left(\Psi^{(0)}\right) \int_{0}^{\eta} \Psi_{Y}^{(0)} d \eta\right]+\mathscr{H}\left(\Psi^{(0)}\right) .
$$

If we integrate (30) between $\eta=0$ and $\eta=2 \pi$ and use periodicity, we are left with

$$
\frac{\partial}{\partial \Psi^{(0)}}\left[\mathscr{F}^{(3) \prime}\left(\Psi^{(0)}\right) \int_{0}^{2 \pi} \Psi_{Y}^{(0)} d \eta\right]=a,
$$

where $a$ is a constant. If we integrate this with respect to $\Psi^{(0)}$, we obtain

$$
\mathscr{F}^{(3) \prime}\left(\Psi^{(0)}\right) \int_{0}^{2 \pi} \Psi_{Y}^{(0)} d \eta=a \Psi^{(0)}+b,
$$

where $b$ is a second constant. This equation (33) holds for all values of $\Psi^{(0)}$, and in particular, we can evaluate it for large values of $\Psi^{(0)}$, which correspond to large values of $|Y|$. In this limit, we know $\mathscr{F}^{(3)}$ from (25), while

$$
\begin{aligned}
\Psi_{Y_{ \pm}}^{(0) \pm}= & \mp \frac{4 Y_{ \pm}}{3^{3 / 2}}= \pm \gamma \frac{2^{3 / 2}}{3^{3 / 4}} \\
& \times\left[\mp \Psi^{(0) \pm}+\left(\frac{2^{1 / 2} A_{1} e^{i \eta}}{3} \pm \frac{2 A_{2} e^{2 i \eta}}{3}+c c\right)\right]^{1 / 2}, \\
\int_{0}^{2 \pi} \Psi_{Y_{ \pm}}^{(0) \pm} d \eta= \pm \gamma \frac{2^{3 / 2}}{3^{3 / 4}} & \\
& \times \int_{0}^{2 \pi}\left[\mp \Psi^{(0) \pm}+\left(\frac{2^{1 / 2} A_{1} e^{i \eta}}{3} \pm \frac{2 A_{2} e^{2 i \eta}}{3}+c c\right)\right]^{1 / 2} d \eta \\
\sim & \pm \gamma \frac{2^{5 / 2}}{3^{3 / 4} \pi}\left(\mp \Psi^{(0) \pm}\right)^{1 / 2} \\
& \times\left[1-\left(\frac{A_{1}^{2}}{18}+\frac{A_{2}^{2}}{9}\right)\left(\mp \Psi^{(0) \pm}\right)^{-2}\right. \\
& {\left[\frac{A_{1}^{2} A_{2}}{18}\left(\mp \Psi^{(0) \pm}\right)^{-3}+\cdots\right] . }
\end{aligned}
$$

Using (34) and (25) in (33), we find that $a^{ \pm}= \pm(32 \pi / \sqrt{3})$, while $b=0$. Using these, we can write

$$
\mathscr{F}^{(3) \prime}\left(\Psi^{(0)}\right)=-\frac{a \Psi^{(0)}}{\int_{0}^{2 \pi} \Psi_{Y}^{(0)} d \eta} .
$$

Comparing our two expressions for $\mathscr{F}^{(3) \prime}\left(\Psi^{(0)}\right)$ also tells us that $\phi_{0 c,-1}^{(3) \pm}=\mp 2 A_{1}^{2} A_{2} / 3^{1 / 2}$, so that the singular part of the $\mathcal{O}\left(\varepsilon^{3 / 2}\right)$ mean flow distortion is only present when both waves are present.
We now need an expression for $\int_{0}^{2 \pi} \Psi_{Y}^{(0)} d \eta$. It is straightforward to show that we can write

$$
\begin{aligned}
\int_{0}^{2 \pi} \Psi_{Y_{ \pm}}^{(0) \pm} d \eta= & \pm \gamma \frac{2^{7 / 2}}{3^{3 / 4}}\left[\mp \Psi^{(0) \pm}+\frac{2^{3 / 2} A_{1}}{3}+\frac{4 A_{2}}{3}\right]^{1 / 2} \\
& \times \int_{0}^{\pi / 2}\left[1-\frac{2^{5 / 2} A_{1} \sin ^{2} \eta+8 A_{2} \sin ^{2} 2 \eta}{\mp 3 \Psi^{(0) \pm}+2^{3 / 2} A_{1}+4 A_{2}}\right]^{1 / 2} d \eta
\end{aligned}
$$

This expression simplifies if only one mode is present. When $A_{2}=0$, it reduces to

$$
\begin{aligned}
\int_{0}^{2 \pi} \Psi_{Y_{ \pm}}^{(0) \pm} d \eta= & \pm \gamma \frac{2^{7 / 2}}{3^{3 / 4}}\left[\mp \Psi^{(0) \pm}+\frac{2^{3 / 2} A_{1}}{3}\right]^{1 / 2} \\
& \times \int_{0}^{\pi / 2}\left[1-\frac{2^{5 / 2} A_{1} \sin ^{2} \eta}{\mp 3 \Psi^{(0) \pm}+2^{3 / 2} A_{1}}\right]^{1 / 2} d \eta \\
= & \pm \gamma \frac{2^{7 / 2}}{3^{3 / 4}}\left[\mp \Psi^{(0) \pm}+\frac{2^{3 / 2} A_{1}}{3}\right]^{1 / 2} \\
& \times E\left(\left[\frac{2^{5 / 2} A_{1}}{\mp 3 \Psi^{(0) \pm}+2^{3 / 2} A_{1}}\right]^{1 / 2}\right),
\end{aligned}
$$

where $E$ is the elliptic integral of the second kind [59], but when $A_{1}=0$,

$$
\begin{aligned}
\int_{0}^{2 \pi} \Psi_{Y_{ \pm}}^{(0) \pm} d \eta= & \pm \gamma \frac{2^{7 / 2}}{3^{3 / 4}}\left[\mp \Psi^{(0) \pm}+\frac{4 A_{2}}{3}\right]^{1 / 2} \\
& \times \int_{0}^{2 \pi}\left[1-\frac{8 A_{2} \sin ^{2} \eta}{\mp 3 \Psi^{(0) \pm}+4 A_{2}}\right]^{1 / 2} d \eta \\
= & \pm \gamma \frac{2^{7 / 2}}{3^{3 / 4}}\left[\mp \Psi^{(0) \pm}+\frac{4 A_{2}}{3}\right]^{1 / 2} \\
& \times E\left(\left[\frac{8 A_{2}}{\mp 3 \Psi^{(0) \pm}+4 A_{2}}\right]^{1 / 2}\right) .
\end{aligned}
$$

Using (36), we have

$$
\begin{aligned}
& \mathscr{F}^{(3) \pm \prime}\left(\Psi^{(0) \pm}\right) \\
&=-\frac{a^{ \pm} \Psi^{(0) \pm}}{\int_{0}^{2 \pi} \Psi_{Y_{ \pm}}^{(0) \pm} d \eta} \\
&=\mp \gamma\left(3^{5 / 4} a^{ \pm}\left[\mp 3 \Psi^{(0) \pm}+2^{3 / 2} A_{1}+4 A_{2}\right]^{-1 / 2} \Psi^{(0) \pm}\right) \\
& \times\left(2^{7 / 2} \int_{0}^{\pi / 2}\left[1-\frac{2^{5 / 2} A_{1} \sin ^{2} \eta+8 A_{2} \sin ^{2} 2 \eta}{\mp 3 \Psi^{(0) \pm}+2^{3 / 2} A_{1}+4 A_{2}}\right]^{1 / 2} d \eta\right)^{-1},
\end{aligned}
$$




$$
\begin{aligned}
\mathscr{F}^{(3) \pm}\left(\Psi^{(0) \pm}\right)=\mp \gamma \frac{3^{5 / 4} a^{ \pm}}{2^{7 / 2}} \\
\times \int_{\Psi_{B}^{(0) \pm}}^{\Psi^{(0) \pm}}\left(\left[\mp 3 \Phi+2^{3 / 2} A_{1}+4 A_{2}\right]^{-1 / 2} \Phi d \Phi\right) \\
\quad \times\left(\int_{0}^{\pi / 2}\left[1-\frac{2^{5 / 2} A_{1} \sin ^{2} \eta+8 A_{2} \sin ^{2} 2 \eta}{\mp 3 \Phi+2^{3 / 2} A_{1}+4 A_{2}}\right]^{1 / 2} d \eta\right)^{-1}
\end{aligned}
$$

where $\Psi_{B}^{(0)}$ denotes the contour bounding the core. Using these expressions, we recover

$$
\begin{aligned}
& \Psi_{Y_{ \pm} Y_{ \pm}}^{(3) \pm} \\
& =\Psi_{Y_{ \pm} Y_{ \pm}}^{(3 C) \pm}+\mathscr{F}^{(3) \pm}\left(\Psi^{(0) \pm}\right) \\
& =\Psi_{Y_{ \pm} Y_{ \pm}}^{(3 C)} \mp \gamma \frac{3^{5 / 4} a^{ \pm}}{2^{7 / 2}} \\
& \quad \times \int_{\Psi_{B}^{(0) \pm}}^{\Psi^{(0) \pm}}\left(\left[\mp 3 \Phi+2^{3 / 2} A_{1}+4 A_{2}\right]^{-1 / 2} \Phi d \Phi\right) \\
& \quad \times\left(\int_{0}^{\pi / 2}\left[1-\frac{2^{5 / 2} A_{1} \sin ^{2} \eta+8 A_{2} \sin ^{2} 2 \eta}{\mp 3 \Phi+2^{3 / 2} A_{1}+4 A_{2}}\right]^{1 / 2} d \eta\right)^{-1} .
\end{aligned}
$$

It is straightforward to recover $\Psi_{B}^{(0)}$, the contour bounding the core, which appears as a limit on the integrals in (39) and (40). If we evaluate $\Psi_{\xi}^{(0)}$ on $Y=0$, we find

$$
\Psi_{\xi}^{(0) \pm}=-\frac{2}{3}\left[ \pm 2^{1 / 2} A_{1}+8 A_{2} \cos \xi\right] \sin \xi
$$

which vanishes at $\xi=0$ and $\pi$ together and also at $A_{1}+$ $2^{5 / 2} A_{2} \cos \xi=0$ for the upper critical layer and $A_{1}-$ $2^{5 / 2} A_{2} \cos \xi$ for the lower critical layer. For the upper critical layer, $A_{1}+2^{5 / 2} A_{2} \cos \xi=0$ is the correct root when $A_{2}>0$ and $-2^{5 / 2} A_{2} \leq A_{1} \leq 2^{5 / 2} A_{2}$, with

$$
\begin{aligned}
& \Psi^{(0)+}=-\frac{A_{2}}{12}\left[16+\left(\frac{A_{1}}{A_{2}}\right)^{2}\right], \\
& \int_{0}^{2 \pi} \Psi_{Y_{+}}^{(0)+} d \eta= \frac{2^{1 / 2}}{3^{5 / 4} \sqrt{A_{2}}} \\
& \times \int_{0}^{2 \pi}\left[A_{1}+2^{5 / 2} A_{2} \cos \xi\right] d \eta=-\frac{2^{3 / 2} \pi A_{1}}{3^{5 / 4} \sqrt{A_{2}}}
\end{aligned}
$$

while for the lower critical layer $A_{1}+2^{5 / 2} A_{2} \cos \xi=0$ is the correct root when $A_{2}<0$ and $2^{5 / 2} A_{2} \leq A_{1} \leq-2^{5 / 2} A_{2}$, with

$$
\begin{aligned}
& \Psi^{(0)-}=-\frac{A_{2}}{12}\left[16+\left(\frac{A_{1}}{A_{2}}\right)^{2}\right] \\
& \int_{0}^{2 \pi} \Psi_{Y_{-}}^{(0)-} d \eta= \frac{2^{1 / 2}}{3^{5 / 4} \sqrt{-A_{2}}} \\
& \times \int_{0}^{2 \pi}\left[A_{1}-2^{5 / 2} A_{2} \cos \xi\right] d \eta=\frac{2^{3 / 2} \pi A_{1}}{3^{5 / 4} \sqrt{-A_{2}}}
\end{aligned}
$$

The root $\xi=0$ is the correct root for the upper critical layer when $A_{1} \leq-2^{5 / 2} A_{2}$, with

$$
\begin{gathered}
\Psi^{(0)+}=\frac{2^{3 / 2} A_{1}}{3}+\frac{4 A_{2}}{3}, \\
\int_{0}^{2 \pi} \Psi_{Y_{+}}^{(0)+} d \eta \\
=-\frac{2^{19 / 4}}{3^{5 / 4}} \int_{0}^{\pi / 2} \sin \frac{\xi}{2}\left[2^{5 / 2} A_{2} \cos \frac{\xi}{2}-\left(A_{1}+2^{5 / 2} A_{2}\right)\right]^{1 / 2} d \eta \\
=-\frac{2^{15 / 4}}{3^{5 / 4}}\left[\sqrt{-A_{1}}-\frac{A_{1}+2^{5 / 2} A_{2}}{\sqrt{2^{5 / 2} A_{2}}} \ln \frac{\sqrt{-A_{1}}+\sqrt{2^{5 / 2} A_{2}}}{\sqrt{-A_{1}-2^{5 / 2} A_{2}}}\right] \\
\text { if } A_{2}>0,
\end{gathered}
$$

while it is the correct root for the lower critical layer when $A_{1} \leq 2^{5 / 2} A_{2}$, with

$$
\Psi^{(0)-}=-\frac{2^{3 / 2} A_{1}}{3}+\frac{4 A_{2}}{3},
$$

$$
\begin{aligned}
& \int_{0}^{2 \pi} \Psi_{Y_{-}}^{(0)-} d \eta \\
& =\frac{2^{19 / 4}}{3^{5 / 4}} \int_{0}^{\pi / 2} \sin \frac{\xi}{2}\left[\left(-A_{1}+2^{5 / 2} A_{2}\right)-2^{5 / 2} A_{2} \cos \frac{\xi}{2}\right]^{1 / 2} d \eta \\
& =-\frac{2^{15 / 4}}{3^{5 / 4}}\left[\sqrt{-A_{1}}-\frac{A_{1}-2^{5 / 2} A_{2}}{\sqrt{2^{5 / 2} A_{2}}} \ln \frac{\sqrt{-A_{1}}+\sqrt{2^{5 / 2} A_{2}}}{\sqrt{-A_{1}+2^{5 / 2} A_{2}}}\right] \\
& =-\frac{2^{15 / 4}}{3^{5 / 4}}\left[\sqrt{-A_{1}}-\frac{A_{1}-2^{5 / 2} A_{2}}{\sqrt{2^{5 / 2} A_{2}}} \arctan \sqrt{\frac{2^{5 / 2} A_{2}}{A_{1}}}\right] \\
& \text { if } A_{2}>0 .
\end{aligned}
$$


The root $\xi=\pi$ is the correct root for the upper critical layer when $A_{1} \geq 2^{5 / 2} A_{2}$, with

$$
\begin{gathered}
\Psi^{(0)+}=-\frac{2^{3 / 2} A_{1}}{3}+\frac{4 A_{2}}{3} \\
\int_{0}^{2 \pi} \Psi_{Y_{+}}^{(0)+} d \eta=-\frac{2^{19 / 4}}{3^{5 / 4}} \\
\times \int_{0}^{\pi / 2} \cos \xi\left[\left(A_{1}-2^{5 / 2} A_{2}\right)+2^{5 / 2} A_{2} \cos ^{2} \xi\right]^{1 / 2} d \eta,
\end{gathered}
$$

while it is the correct root for the lower critical layer when $A_{1} \geq-2^{5 / 2} A_{2}$, with

$$
\begin{gathered}
\Psi^{(0)-}=\frac{2^{3 / 2} A_{1}}{3}+\frac{4 A_{2}}{3} \\
\int_{0}^{2 \pi} \Psi_{Y_{-}}^{(0)-} d \eta=\frac{2^{19 / 4}}{3^{5 / 4}} \\
\times \int_{0}^{\pi / 2} \cos \xi\left[\left(A_{1}+2^{5 / 2} A_{2}\right)-2^{5 / 2} A_{2} \cos ^{2} \xi\right]^{1 / 2} d \eta .
\end{gathered}
$$

One last piece of analysis needs to be performed. Inside the core, we have essentially set $\mathscr{F}_{3} \equiv 0$, which means that the gradient of $\mathscr{F}_{3}$ is discontinuous across the core with

$$
\begin{gathered}
\mathscr{F}^{(3)+1} \longrightarrow \begin{cases}\mathscr{F}_{B}^{(3)+1} & \text { as } \Psi^{(0)+} \longrightarrow \Psi_{B}^{(0)+}- \\
0 & \text { as } \Psi^{(0)+} \longrightarrow \Psi_{B}^{(0)+}+,\end{cases} \\
\mathscr{F}^{(3)-1} \longrightarrow \begin{cases}\mathscr{F}_{B}^{(3)-1} & \text { as } \Psi^{(0)-} \longrightarrow \Psi_{B}^{(0)-}+, \\
0 & \text { as } \Psi^{(0)-} \longrightarrow \Psi_{B}^{(0)-}-\end{cases}
\end{gathered}
$$

where $\mathscr{F}_{B}^{(3) \prime}$ can be recovered from the above analysis. Because of this, a thin diffusive layer is necessary at the edge of the core to smooth out this discontinuity; this approach is due to Haberman $[23,24]$. We will pose a new inner expansion of $\Psi$ in $\varepsilon$, retaining the viscous terms as follows:

$$
\begin{aligned}
\Psi^{(v)} \sim & \Psi^{(0 v)}+\varepsilon^{1 / 2} \Psi^{(1 v)}+\varepsilon \Psi^{(2 v)} \\
& +\varepsilon^{3 / 2}\left[\Psi^{(3 v)}+\left(\ln \varepsilon^{1 / 2}\right) \Psi^{(3 l v)}\right]+\cdots
\end{aligned}
$$

Since we have already shown that the $\mathcal{O}\left(\varepsilon^{0}\right), \mathcal{O}\left(\varepsilon^{1 / 2}\right)$, and $\mathcal{O}\left(\varepsilon^{1}\right)$ viscous corrections all vanish, $\widetilde{\Psi}^{(0)}=\widetilde{\Psi}^{(1)}=\widetilde{\Psi}^{(2)}=0$, we can deduce that, at those orders, the viscous and inviscid solutions are identical, so that $\Psi^{(0 v)}=\Psi^{(0)}, \Psi^{(1 v)}=\Psi^{(1)}$, and $\Psi^{(2 v)}=\Psi^{(2)}$. At $\mathcal{O}\left(\varepsilon^{3 / 2}\right)$, we will write $\Psi^{(3 v)}=\Psi^{(3 C v)}+\Psi^{(3 D v)}$, with $\Psi^{(3 C v)}=\Psi^{(3 C)}$, while $\Psi^{(3 D v)}$ obeys

$$
\frac{\partial\left(\Psi^{(0)}, \Psi_{Y Y}^{(3 D v)}\right)}{\partial(\xi, Y)}=-\lambda \Psi_{Y Y Y Y}^{(3 D v)}
$$

or writing $\Theta=\Psi_{Y Y}^{(3 D v)}$,

$$
\frac{\partial\left(\Psi^{(0)}, \Theta\right)}{\partial(\xi, Y)}=-\lambda \Theta_{Y Y}
$$

A similar equation to this was found in [20], and we can employ the solution technique employed there. Once again we will use $\eta=\xi$ and $\Psi^{(0)}$ as von Mises coordinates, and to leading order, we can write (51) as

$$
\begin{aligned}
\Psi_{Y}^{(0)} \Theta_{\eta} & =\lambda\left(\Psi_{Y}^{(0) 2} \Theta_{\Psi^{(0)} \Psi^{(0)}}+\Psi_{Y Y}^{(0)} \Theta_{\Psi^{(0)}}\right) \\
& =\lambda\left(\Psi_{Y}^{(0) 2} \Xi_{\Psi^{(0)}}+u_{0 c}^{\prime} \Xi\right),
\end{aligned}
$$

or

$$
\Psi_{Y_{ \pm}}^{(0) \pm} \Theta_{\eta}^{ \pm}=\lambda\left(\Psi_{Y_{ \pm}}^{(0) \pm 2} \Xi_{\Psi^{(0) \pm}}^{ \pm} \mp \frac{4 \Xi^{ \pm}}{3^{3 / 2}}\right),
$$

where we have defined $\Xi=\Theta_{\Psi^{(0)}}$. Since we want the diffusive and nonlinear terms to balance inside the diffusive layer, we will introduce the rescaled stream function $\Upsilon=\lambda^{-1 / 2} \Psi^{(0)}$, and then

$$
\Theta_{\eta}=\lambda^{1 / 2}\left[\Psi_{Y}^{(0)} \Xi_{\Upsilon}+\mathcal{O}\left(\lambda^{1 / 2}\right)\right]
$$

or differentiating this with respect to $\Upsilon$,

$$
\Xi_{\eta}=\Psi_{Y}^{(0)} \Xi_{\Upsilon Y}+\mathcal{O}\left(\lambda^{1 / 2}\right),
$$

so that

$$
\Xi_{\eta}^{ \pm} \approx \pm \gamma \frac{4}{3^{5 / 4}}\left(2^{-1 / 2} A_{1} \cos \eta \pm A_{2} \cos 2 \eta\right)^{1 / 2} \Xi_{\Upsilon \Upsilon}^{ \pm} .
$$

This has a solution

$$
\begin{aligned}
& \Xi^{ \pm} \\
& \mathscr{F}_{B}^{(3) / \pm} \operatorname{erf}\left(\frac{3^{5 / 8}\left(\Upsilon^{ \pm}-\Upsilon_{B}^{ \pm}\right)}{2^{3 / 2}\left(\sqrt{2} A_{1}(1 \pm \sin \xi)+A_{2}(1 \pm \sin 2 \xi)\right)^{1 / 2}}\right) \\
& \mathscr{F}_{B}^{(3) / \pm} \operatorname{erf}\left(\frac{Y_{ \pm}>0}{3^{5 / 8}\left(\Upsilon^{ \pm}-\Upsilon_{B}^{ \pm}\right)}\right) \\
& Y_{ \pm}<0 .
\end{aligned}
$$

Having found $\Xi$, we can recover $\Theta=\Psi_{Y Y}^{(3 D v)}$ using (54), meaning that it is possible to smooth out the discontinuity in the gradient of $\mathscr{F}^{(3)}$. This completes our analysis.

Our results are shown graphically in Figure 1 where we plot the inner stream function. In Figures 1(a) and 1(b), we show the flow in the upper and lower critical layers, respectively, when only $A_{1}$ is present, and the two critical layers are in phase with one vortex present in the box $0 \leq \xi \leq$ $2 \pi$. In Figures $1(\mathrm{c})$ and $1(\mathrm{~d})$, we show the flow when only $A_{2}$ is present, and the two critical layers are out of phase with two vortices present in $0 \leq \xi \leq 2 \pi$. In each of Figures 1(a) $-1(d)$, the flow in either critical layer looks like a row of Stuart 


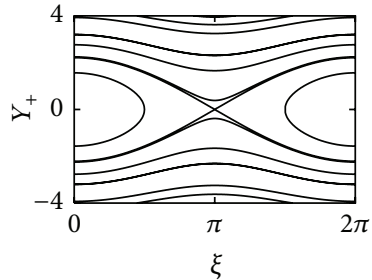

(a)

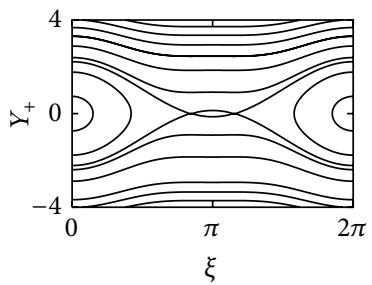

(e)

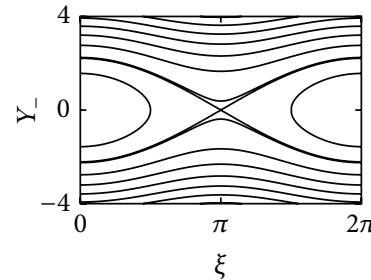

(b)

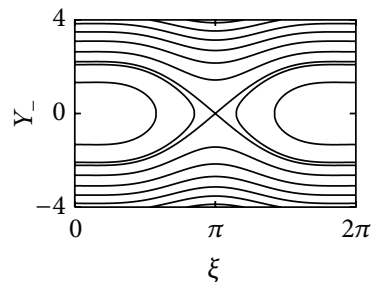

(f)

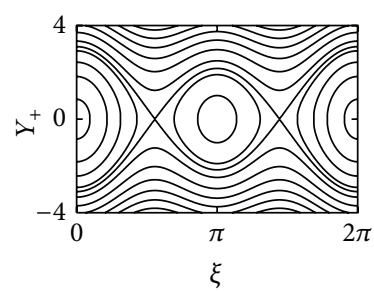

(i)

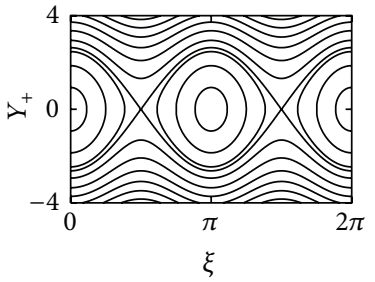

(c)

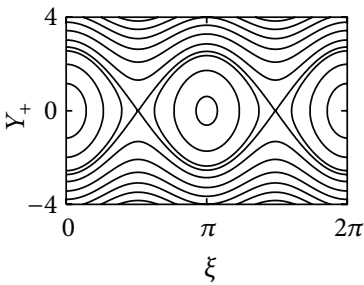

(g)

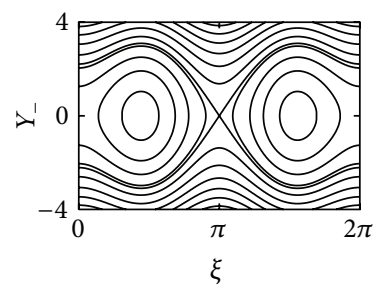

(j)

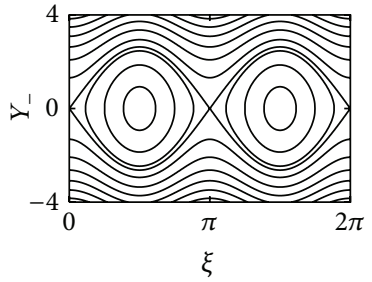

(d)

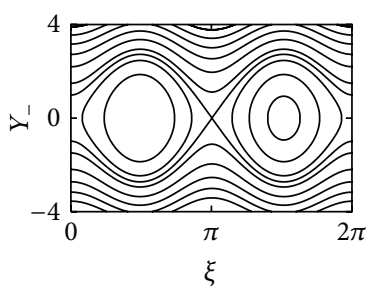

(h)

Figure 1: (a) $A_{1}=1, A_{2}=0$, upper critical layer; (b) $A_{1}=1, A_{2}=0$, lower critical layer; (c) $A_{1}=0, A_{2}=1$, upper critical layer; (d) $A_{1}=0$, $A_{2}=1$, lower critical layer; (e) $A_{1}=1, A_{2}=0.2$, upper critical layer; (f) $A_{1}=1, A_{2}=0.2$, lower critical layer; (g) $A_{1}=0.2, A_{2}=1$, upper critical layer; (h) $A_{1}=0.2, A_{2}=1$, lower critical layer; (i) $A_{1}=1, A_{2}=1$, upper critical layer; (j) $A_{1}=1, A_{2}=1$, lower critical layer.

vortices. In the remaining figures, both modes are present. In Figures 1(e) and 1(f), $A_{1} \gg A_{2}$ and the flow resembles that in Figures 1(a) and 1(b). In Figures 1(g) and 1(h), $A_{2} \gg A_{1}$ and the flow resembles that in Figures 1(c) and 1(d). In Figures $1(\mathrm{i})$ and $1(\mathrm{j})$, the two modes are of comparable size and the two critical layers differ from each other slightly. In the upper layer, one vortex is larger than the other, while in the lower layer, the two vortices are of comparable size but are starting to merge at the edge of the box.

\section{Discussion}

In the previous section, we have used nonlinear critical layer techniques to find nonlinear quasi-steady-state vortex solutions for the plane jet; these solutions represent the quasiequilibrium large scale vortices rings that emerge following vortex rollup in a plane jet. These solutions have a smooth but nonuniform vorticity distribution inside the cores, so they may be thought of as Stuart vortices in a plane jet, rather than BBD-style vorticity-homogenized cores $[13,14]$. Our reasons for using the Stuart vortex as the solution inside the core were presented in the introduction and are essentially due to a combination of the Brown and Stewartson argument that the cat's eyes would decay before vorticity has had time to homogenize [35], which has been confirmed by numerical simulations and the claim by Sommeria et al. [42] that the Stuart vortex is a preferred state on equilibrium grounds.

\section{Conflict of Interests}

The authors declare that there is no conflict of interests regarding the publication of this paper.

\section{Acknowledgments}

The authors would like to thank the referees for their insightful suggestions and comments which greatly improved the paper, Roger Grimshaw and Philippe Caillol for generously providing copies of their work, and Philippe Caillol for his insightful remarks on whether a steady solution would be attained in practice.

\section{References}

[1] G. E. Mattingly and W. O. Criminale, "The stability of an incompressible two-dimensional wake," Journal of Fluid Mechanics, vol. 51, no. 2, pp. 233-272, 1972.

[2] P. Savic, "On acoustically effective vortex motion in gaseous jets," Philosophical Magazine, vol. 32, pp. 245-252, 1941.

[3] P. Savic and J. W. Murphy, "The symmetrical vortex street in sound sensitive plane jets," Philosophical Magazine, vol. 34, pp. 
139-144, 1943.

[4] L. N. Howard and P. G. Drazin, "On instability of a parallel flow of inviscid fluid in a rotating system with variable Coriolis parameter," Journal of Mathematical Physics, vol. 43, pp. 83-99, 1964.

[5] R. Mallier and A. M. J. Davis, "Nonlinear evolution of singular disturbances to the Bickley jet," Studies in Applied Mathematics, vol. 103, no. 2, pp. 149-181, 1999.

[6] G. E. Swaters, "On the evolution of near-singular modes of the Bickley jet," Physics of Fluids, vol. 11, no. 9, pp. 2546-2555, 1999.

[7] R. E. Kelly, "On the resonant interaction of neutral disturbances in two inviscid shear flows," Journal of Fluid Mechanics, vol. 31, no. 4, pp. 789-799, 1968.

[8] I. Wygnanski, F. Champagne, and B. Marasli, "On the large scale structures in two dimensional, small deficit turbulent wakes," Journal of Fluid Mechanics, vol. 168, pp. 31-71, 1986.

[9] H. Sato, "An experimental study of nonlinear interaction of velocity fluctuations in the transition region of a twodimensional wake," Journal of Fluid Mechanics, vol. 44, no. 4, pp. 741-765, 1970.

[10] H. Sato and H. Saito, "Artificial control of laminar-turbulent transition of a two-dimensional wake by external sound," Journal of Fluid Mechanics, vol. 84, no. 4, pp. 657-672, 1978.

[11] R. Mallier and M. C. Haslam, "Modal interactions in a Bickley jet: comparison of theory with direct numerical simulation," Mathematics and Computers in Simulation, vol. 62, no. 1-2, pp. 109-115, 2003.

[12] S. J. Leib and M. E. Goldstein, "Nonlinear interaction between the sinuous and varicose instability modes in a plane wake," Physics of Fluids A, vol. 1, no. 3, pp. 513-521, 1989.

[13] D. J. Benney and R. T. Bergeron, "A new class of nonlinear waves in parallel flows," Studies in Applied Mathematics, vol. 48, no. 3, pp. 181-204, 1969.

[14] R. E. Davis, "On the high Reynolds number flow over a wavy boundary," Journal of Fluid Mechanics, vol. 36, no. 2, pp. 337$346,1969$.

[15] T. Warn and H. Warn, "The evolution of a nonlinear critical layer," Studies in Applied Mathematics, vol. 59, no. 1, pp. 37-71, 1978.

[16] K. Stewartson, "The evolution of the critical layer of a Rossby wave," Geophysical and Astrophysical Fluid Dynamics, vol. 9, pp. 185-200, 1978.

[17] R. Mallier, "Fully coupled resonant triad interactions in a Bickley jet," European Journal of Mechanics B, vol. 15, no. 4, pp. 507-520, 1996.

[18] R. Mallier and M. C. Haslam, "Interactions between pairs of oblique waves in a Bickley jet," European Journal of Mechanics $B$, vol. 18 , no. 2 , pp. 227-243, 1999.

[19] J. T. Stuart, "On finite amplitude oscillations in laminar mixing layers," Journal of Fluid Mechanics, vol. 29, no. 3, pp. 417-440, 1967.

[20] R. E. Kelly and S. A. Maslowe, "Nonlinear critical layer in a slightly stratified shear flow," Studies in Applied Mathematics, vol. 49, no. 4, pp. 301-332, 1970.

[21] S. A. Maslowe, "The generation of clear air turbulence by nonlinear waves," Studies in Applied Mathematics, vol. 51, no. 1, pp. 1-16, 1972.

[22] S. A. Maslowe, "Finite-amplitude Kelvin-Helmholtz billows," Boundary-Layer Meteorology, vol. 5, no. 1-2, pp. 43-52, 1973.

[23] R. Haberman, "Critical layers in parallel flows," Studies in Applied Mathematics, vol. 51, no. 2, pp. 139-161, 1972.
[24] R. Haberman, "Wave-induced distortions of a slightly stratified shear flow: nonlinear critical-layer effect," Journal of Fluid Mechanics, vol. 58, no. 4, pp. 727-735, 1973.

[25] G. P. Klaassen and W. R. Peltier, "Role of transverse secondary instabilities in the evolution of free shear layers," Journal of Fluid Mechanics, vol. 202, pp. 367-402, 1989.

[26] R. T. Pierrehumbert and S. E. Widnall, "The two- and threedimensional instabilities of a spatially periodic shear layer," Journal of Fluid Mechanics, vol. 114, pp. 59-82, 1982.

[27] S. Leblanc and C. Cambon, "Effects of the Coriolis force on the stability of Stuart vortices," Journal of Fluid Mechanics, vol. 356, pp. 353-379, 1998.

[28] G. Alobaidi, C. J. Smith, and R. Mallier, "Waves on a Stuart vortex," Applied Mathematics and Computation, vol. 227, pp. 370-383, 2014

[29] P. Caillol and S. A. Maslowe, "The small vorticity nonlinear critical layer for Kelvin modes on a vortex," Studies in Applied Mathematics, vol. 118, no. 3, pp. 221-254, 2007.

[30] P. Caillol, "Multiple vortices induced by a tridimensional critical layer in a rapidly rotating vortex," IMA Journal of Applied Mathematics, vol. 77, no. 3, pp. 282-292, 2012.

[31] P. Caillol and R. Grimshaw, "Steady multipolar planar vortices with nonlinear critical layers," Geophysical and Astrophysical Fluid Dynamics, vol. 98, no. 6, pp. 473-506, 2004.

[32] R. Mallier and S. A. Maslowe, "A row of counter-rotating vortices," Physics of Fluids A, vol. 5, no. 4, pp. 1074-1075, 1992.

[33] A. Barcilon and P. G. Drazin, "Nonlinear waves of vorticity," Studies in Applied Mathematics, vol. 106, no. 4, pp. 437-479, 2001.

[34] G. K. Batchelor, "On steady laminar flow with closed streamlines at large Reynolds numbers," Journal of Fluid Mechanics, vol. 1, no. 2, pp. 177-190, 1956.

[35] S. N. Brown and K. Stewartson, "The evolution of the critical layer of a Rossby wave. Part II," Geophysical and Astrophysical Fluid Dynamics, vol. 10, no. 1, pp. 1-18, 1978.

[36] K. Stewartson, “Theory of nonlinear stability," Fluid Dynamics Transactions, vol. 7, pp. 101-120, 1974.

[37] L. M. Mack, "A numerical study of the temporal eigenvalue spectrum of the Blasius boundary layer," Journal of Fluid Mechanics, vol. 73, no. 3, pp. 497-520, 1976.

[38] J. W. Murdock and K. Stewartson, "Spectra of the OrrSommerfeld equation," Physics of Fluids, vol. 20, no. 9, pp. 14041411, 1977.

[39] P. C. Patnaik, F. S. Sherman, and G. M. Corcos, "A numerical study of Kelvin-Helmholtz waves of finite amplitude," Journal of Fluid Mechanics, vol. 73, no. 2, pp. 215-240, 1976.

[40] G. P. Klaassen and W. R. Peltier, "The effect of Prandtl number on the evolution and stability of Kelvin-Helmholtz billows," Geophysical and Astrophysical Fluid Dynamics, vol. 32, no. 1, pp. 23-60, 1985.

[41] G. P. Klaassen and W. R. Peltier, "Influence of stratification on secondary instability in free shear layers," Journal of Fluid Mechanics, vol. 227, pp. 71-106, 1991.

[42] J. Sommeria, C. Staquet, and R. Robert, "Final equilibrium state of a two-dimensional shear layer," Journal of Fluid Mechanics, vol. 233, pp. 661-689, 1991.

[43] L. P. Wang and M. R. Maxey, "Kinematical descriptions for mixing in stratified or homogeneous shear flows," in Mixing in Geophysical Flows, J. M. Redondo and O. Métais, Eds., pp. 1434, CINME, Barcelona, Spain, 1995. 
[44] P. B. Rhines and W. R. Young, "How rapidly is a passive scalar mixed within closed streamlines?" Journal of Fluid Mechanics, vol. 133, pp. 133-145, 1983.

[45] P. D. Killworth and M. E. McIntyre, "Do Rossby-wave critical layers absorb, reflect, or over-reflect?" Journal of Fluid Mechanics, vol. 161, pp. 449-492, 1985.

[46] P. H. Haynes, "Nonlinear instability of a Rossby-wave critical layer," Journal of Fluid Mechanics, vol. 161, pp. 493-511, 1985.

[47] P. H. Haynes, "The effect of barotropic instability on the nonlinear evolution of a Rossby-wave critical layer," Journal of Fluid Mechanics, vol. 207, pp. 231-266, 1989.

[48] P. Caillol and R. H. Grimshaw, "Internal solitary waves with a weakly stratified critical layer," Physics of Fluids, vol. 24, no. 5, Article ID 056602, 2012.

[49] P. Caillol and R. H. Grimshaw, "Rossby solitary waves in the presence of a critical layer," Studies in Applied Mathematics, vol. 118, no. 4, pp. 313-364, 2007.

[50] P. Caillol and R. H. Grimshaw, "Rossby elevation waves in the presence of a critical layer," Studies in Applied Mathematics, vol. 120, no. 1, pp. 35-64, 2008.

[51] Y. I. Troitskaya, "Viscous-diffusion nonlinear critical layer in a stratified shear flow," Journal of Fluid Mechanics, vol. 233, pp. 25-48, 1991.

[52] Y. I. Troitskaya and S. N. Reznik, "Quasi-steady dissipative nonlinear critical layer in a stratified shear flow," Physics of Fluids, vol. 8, no. 12, pp. 3313-3328, 1996.

[53] R. Mallier, "Stuart vortices in a stratified mixing layer. I: the Garcia model," Geophysical and Astrophysical Fluid Dynamics, vol. 74, no. 1-4, pp. 73-97, 1994.

[54] R. Mallier, "Stuart vortices on a beta-plane," Dynamics of Atmospheres and Oceans, vol. 22, no. 4, pp. 213-238, 1995.

[55] R. Mallier, "Stuart vortices in a stratified mixing layer: the Holmboe model," Journal of Engineering Mathematics, vol. 47, no. 2, pp. 121-136, 2003.

[56] T. B. Gatski, "Vortex motion in a wall-bounded viscous flow," Proceedings of The Royal Society of London A, vol. 397, no. 1813, pp. 397-414, 1985.

[57] D. I. Meiron, D. W. Moore, and D. I. Pullin, "On steady compressible flows with compact vorticity; the compressible Stuart vortex," Journal of Fluid Mechanics, vol. 409, pp. 29-49, 2000.

[58] G. O'Reilly and D. I. Pullin, "Structure and stability of the compressible Stuart vortex," Journal of Fluid Mechanics, no. 493, pp. 231-254, 2003.

[59] I. S. Gradšteĭn and I. M. Ryžik, Tablitsy Integralov, Summ, Ryadov i Proizvedenii, Gosudarstvennoe Izdatel'stvo FizikoMatematicheskoy Literatury, Moscow, Russia, 1963. 


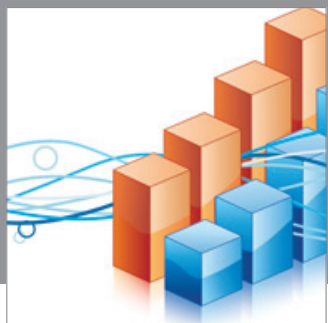

Advances in

Operations Research

mansans

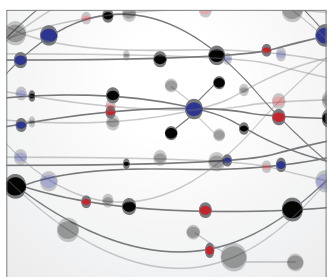

The Scientific World Journal
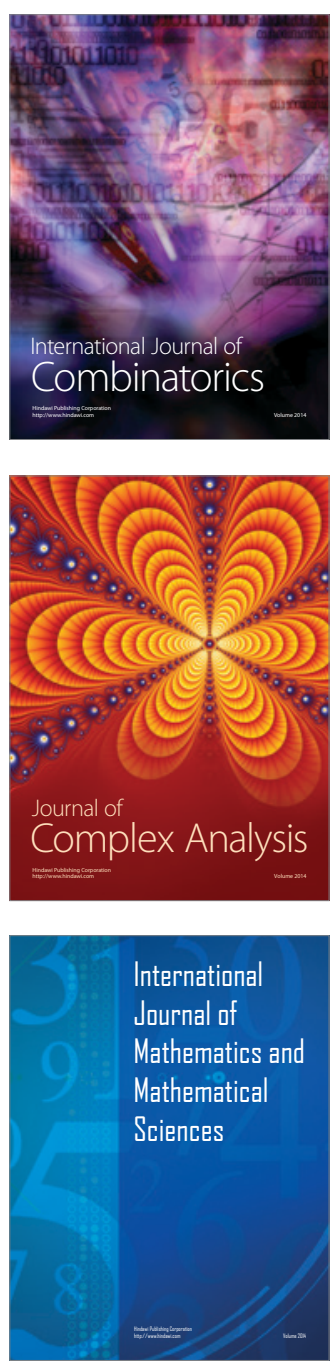
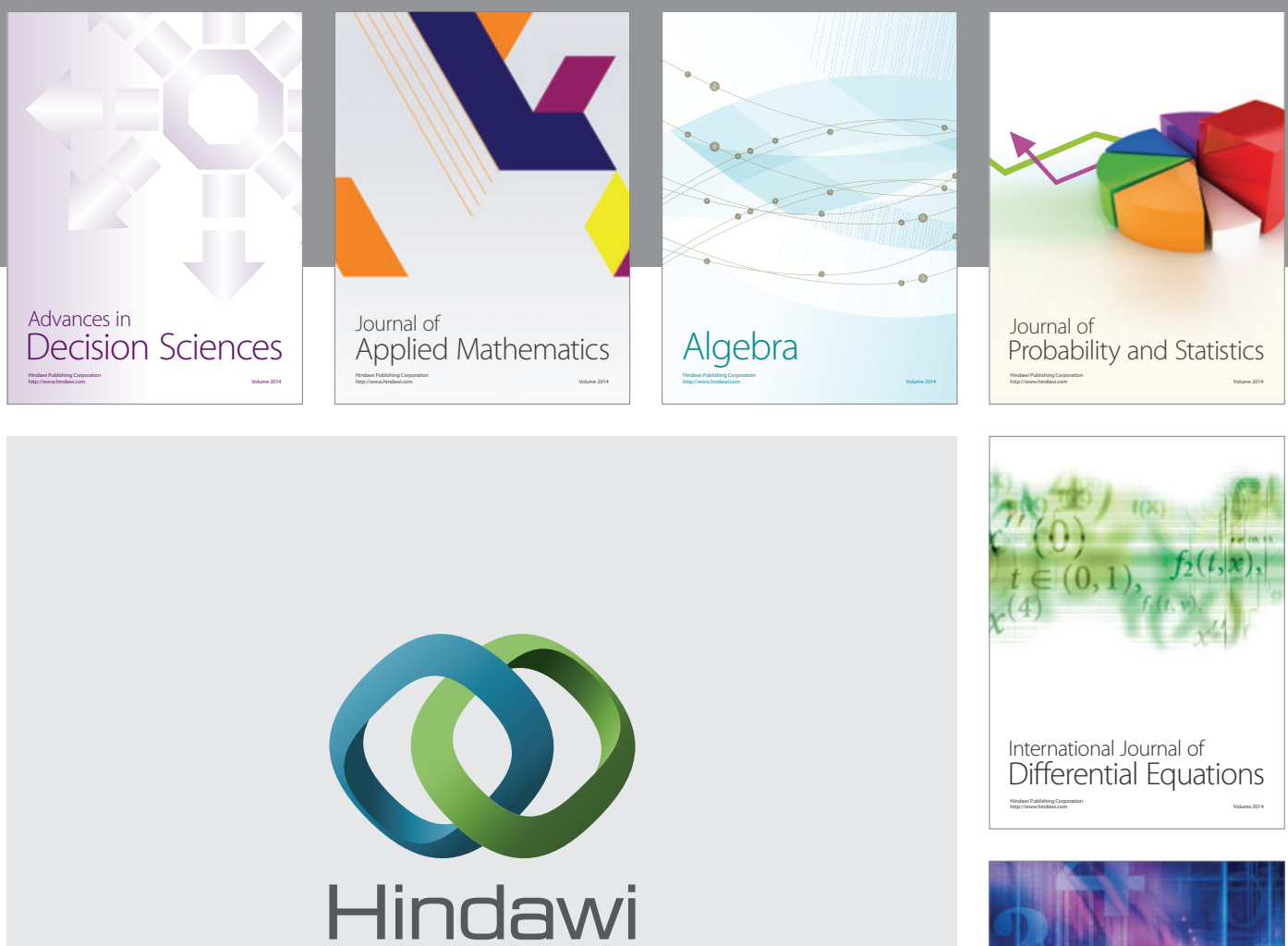

Submit your manuscripts at http://www.hindawi.com
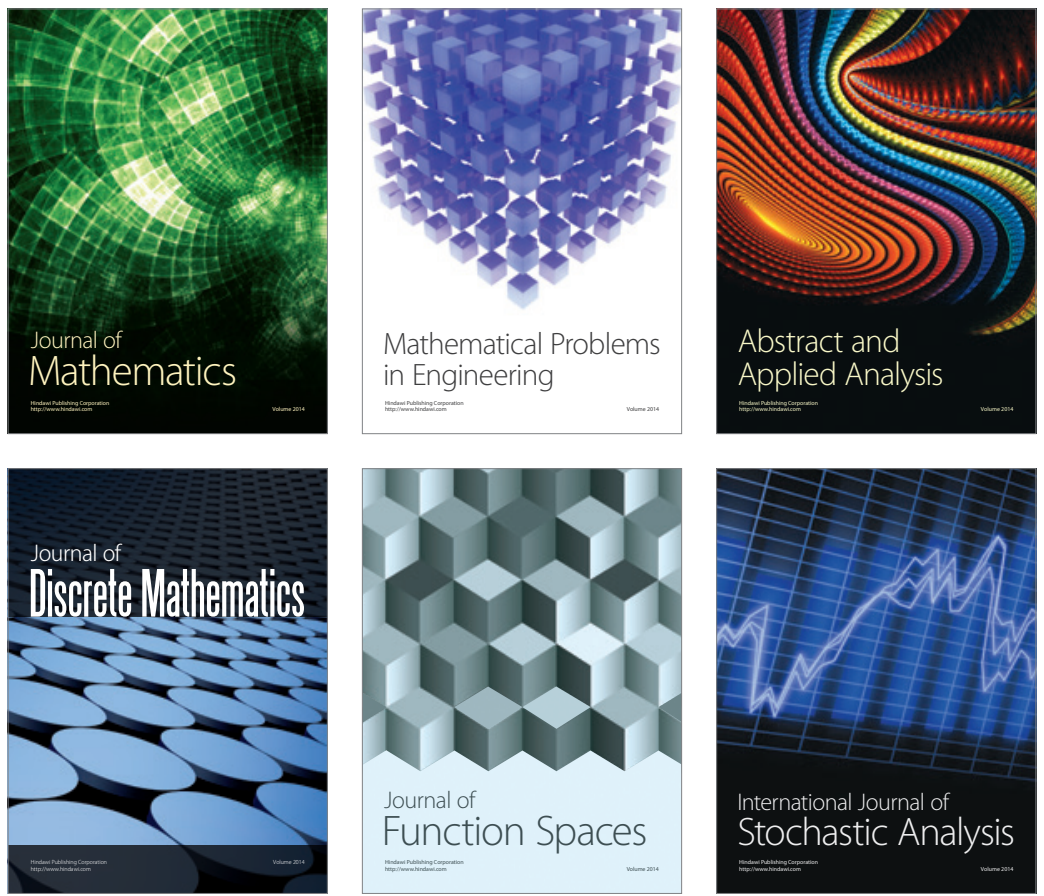

Journal of

Function Spaces

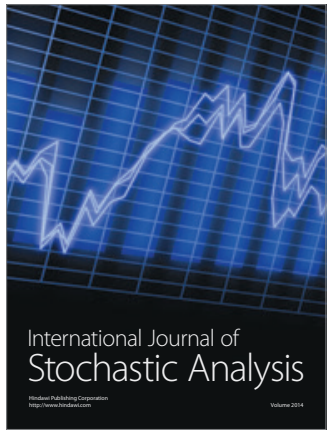

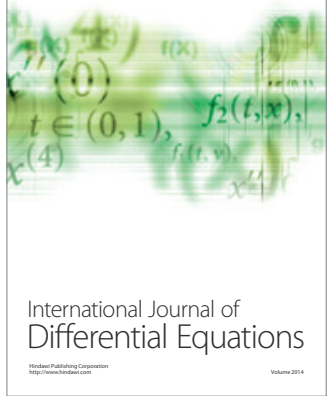
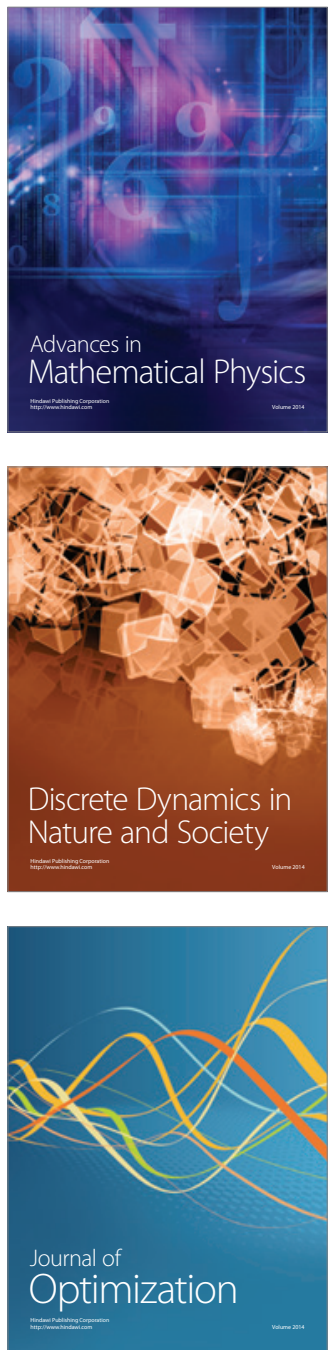